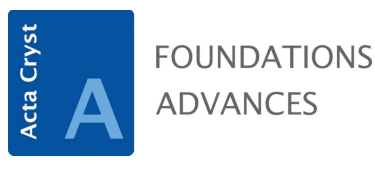

ISSN 2053-2733

\title{
International Tables for Crystallography, Volume A, Space-group symmetry. 6th edition. Edited by Mois I. Aroyo. Wiley, 2016. Pp. xxi + 873. Price GBP 295.00, EUR 354.00 (hardcover). ISBN 978-0-470-97423-0.
}

\author{
Massimo Nespolo* \\ Université de Lorraine, CRM2, UMR 7036, Vandoeuvre-les-Nancy, F-54506, France, and CNRS, CRM2, UMR 7036, \\ Vandoeuvre-les-Nancy, F-54506, France. *Correspondence e-mail: massimo.nespolo@univ-lorraine.fr
}

Keywords: book review; International Tables for Crystallography; space-group symmetry.

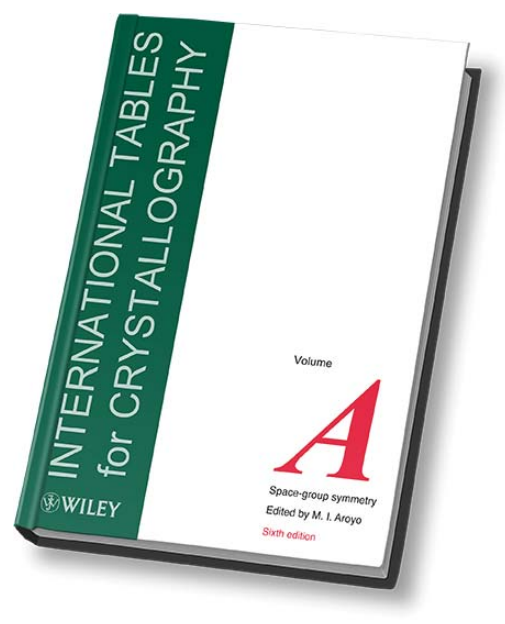

(C) 2017 International Union of Crystallography
Volume A of International Tables for Crystallography approaches its 100th birthday. In fact, although it first appeared under this title only in 1935, its predecessor is the book by Wyckoff (1922), which already contained lists of general and special equivalent positions (later to be called Wyckoff positions) and, in its second edition (Wyckoff, 1930), also three-dimensional drawings of the symmetry elements within the (conventional) unit cells of most of the space-group types.

This brand-new edition of Volume A looks smaller than the previous one, despite the almost identical number of pages (873 versus 911 ); this has been achieved by using a glossy paper which is thinner but no less robust than that for the previous edition. The corresponding decrease in weight is a side effect that will certainly be appreciated by the reader. The sixth edition presents many changes, and many improvements, with respect to the fifth edition (denoted ITA5 in the following), which is now 15 years old (although minor corrections have been published in the meantime). One of the most significant improvements is the introductory material in Part 1, where, using the matrix formalism, the authors have made a remarkable effort to offer a homogeneous text of educational nature explaining the different kinds of symmetry information found in the tables.

The daily user of crystallographic techniques is certainly most familiar with (and interested in) the space-group tables. We therefore start our analysis with the changes in this part of the volume. The most evident difference is in the cubic space-group types, where the diagrams of the general positions have been completely redrawn using VESTA (Momma \& Izumi, 2011) and are now readable and understandable. For the holohedral groups, the general-position diagrams are now also shown in perspective projection (in the online edition these diagrams are shown for all cubic groups). Another change is the disappearance of the lists of maximal subgroups and minimal supergroups; these lists were incomplete and therefore potentially misleading for the inexperienced user. The complete information is today available in Volume A1 (Wondratschek \& Müller, 2010) so there is no reason to keep a partial, highly incomplete version in Volume A. In ITA5, each space-group type was presented over two pages (with a few exceptions for some hexagonal and cubic cases, which spanned over three pages): the diagrams and the information about the origin, asymmetric unit and symmetry operations on the left, the rest on the right. In the new edition, the removal of the incomplete group-subgroup information has enabled some groups to be presented on only one page.

More fundamental and profound changes in this new edition concern the theoretical chapters, which seem to be insufficiently known to and read by 'daily' crystallographers. As a matter of fact, the volumes in the series International Tables for Crystallography provide much more than tables and diagrams: they are the authoritative reference for each and every aspect of our discipline and contain the precise and exact definitions of every term and concept, accompanied by a wide range of examples. In ITA5, a few sections required some special effort by the reader, especially the newcomer to the field; this has been largely taken into account in the new edition, thanks also to new authors joining the team. The material has been considerably reorganized and some parts of the text have been completely rewritten, making them definitely more approachable while 
keeping the rigour that is mandatory in a reference text. We therefore proceed in our critique by making comparisons with ITA5.

The sixth edition has been reorganized into three parts: (1) Introduction to space-group symmetry; (2) The space-group tables; and (3) Advanced topics on space-group symmetry. Although at first sight this may seem to closely follow the structure of ITA5, with just some merging into a single part of what were separate parts, in fact some of the topics that were previously after the space-group tables, and thus implicitly considered to be 'advanced topics', now come before and have been largely rewritten. This is the case for Part 8 of ITA5 (Introduction to space-group symmetry), where basic definitions as well as fundamental concepts like crystal classes, crystal families, crystal systems and lattice systems, Wyckoff positions, crystallographic orbits, generators and normalizers are introduced. These topics now span Chapters 1.3 and 1.4, and have been extensively rewritten in a more reader-friendly way.

Part 1 bears the title Introduction to space-group symmetry and is divided into seven chapters. The first chapter, $A$ general introduction to groups, is a new and most welcome addition. Authored by B. Souvignier, a rare example of a mathematician who is capable of conciliating the necessary rigour with a language close to that used by the daily crystallographer, this chapter concentrates in ten pages the basic definitions of groups, subgroups, cosets, homo- and isomorphisms and related concepts. It stands as the foundation of the whole construction that follows and should be digested by everybody who wants to go beyond the surface. Chapter 1.2 is devoted to the introduction and definition of symmetry operations and their matrix representation, symmetry elements and geometric elements. We find here part of the contents of Chapter 8.1 and Part 11 of ITA5, largely rewritten to give a more consistent and easy-to-follow presentation. The choice of moving this content to before, rather than after, the space-group tables is definitely wise.

Chapter 1.3 is A general introduction to space groups. Starting from the concepts of lattices and unit cells, it introduces the definitions of point and space groups as well as their classification into classes, systems and families. This content has been moved from Part 8 in ITA5 and rewritten, lifting what several readers experienced as a fog; now there is really no further excuse to mistake trigonal, rhombohedral and hexagonal (just to give an example): everything is crystal (sic!) clear.

Chapter 1.4 is devoted to a detailed presentation and explanation of space-group symbols, operations (including the Seitz symbols that were not used in ITA5, although they were already used in Volume E), the construction and interpretation of space-group diagrams, the choice of the generators, Wyckoff positions, Wyckoff sets and crystallographic orbits, and is completed by a few pages on sections and projections of space groups. Parts of the content of this chapter were previously scattered across different sections of ITA5 (e.g. Part 11) but most of the text is new or completely rewritten. This chapter is an absolute must-read for everybody who wants to be able to extract the wealth of information contained in the space-group tables. The text, accompanied by a number of examples (as is the case for all the chapters), is remarkably well written and easy to follow.

Chapter 1.5 deals with the transformation of coordinate systems. The first part of this chapter corresponds to the fifth chapter (Transformations in crystallography) of ITA5, with some additions, redrawn figures and new examples. The second part, about transformations of space-group descriptions, is brand new and is certainly of great help to the reader who is too often at risk of losing their way when staring at nonconventional symbols. The third part of the chapter contains the synoptic tables of plane and space groups. With respect to Part 4 of ITA5, where the same tables were presented, we note two differences: (1) a completely rewritten and enlarged theoretical introduction, and (2) a more consistent notation for the extended symbols of space groups. In fact, while the short and full Hermann-Mauguin symbols of space groups present the symmetry elements along each symmetry direction, the extended symbols give the symmetry operations. For space groups containing the double glide plane (symbol $e$ ) the extended symbols should give the two glides (operations) instead of writing the glide plane (element) twice, as was the case in ITA5. This inconsistency has now been fixed. The tables are still partly incomplete, because for monoclinic groups the settings corresponding to a non-conventional unit cell with double volume ( $S$ instead of $P ; F$ instead of $C$ ) are absent. These are, however, present in the literature (Nespolo \& Aroyo, 2016) and may perhaps be included in a future revision.

Chapter 1.6 is devoted to methods of space-group determination. We find the tables presenting the reflection conditions compatible with the various types of space groups (Part 3 in ITA5), now preceded by a detailed theoretical introduction about the information that can be obtained from diffraction data (this part makes a useful link with Volume B) and followed by guidelines for space-group determination in macromolecular crystallography, from powder diffraction and from electron microscopy. A wealth of information has been added with respect to ITA5. It is regrettable, however, that the extinction symbol has been removed. Apart from the bad choice of name, which is reminiscent of the incorrect yet often used term 'systematic extinctions', this symbol is widely used, for example in electron crystallography and in the analysis of the diffraction patterns of twins. The information needed to build the symbol is all present, but the handy and useful symbol is gone. We hope that it will be reinstated in the first reprint, whenever it comes out.

Chapter 1.7 is a short introduction to Volumes A1 and E, and is a very welcome addition that should help the reader to understand that Volume A, although extremely rich in information, is not the end of the story. Examples of groupsubgroup relations, derivative structures, phase transitions, Bärnighasen trees, subperiodic groups and domain walls are presented. After reading this appetizer, the reader will certainly rush to discover a whole world beneath the usual three-periodic landscape of stand-alone structures. 
This chapter also supersedes Part 13 of ITA5 (Isomorphic subgroups of space groups).

Part 2, as we have mentioned, contains the well known tables of space groups. The theoretical introduction that came at the very beginning of ITA5 is now right before the tables, a much more reasonable choice especially for the occasional user.

Part 3 (Advanced topics on space-group symmetry) is divided into six chapters and includes the revised content of Parts 9, 10, 14 and 15 of ITA5 (the content of Parts 11 and 13 having been moved to Part 1). The text has been revised, corrected, updated and in some parts rewritten with new and extended content.

Chapter 3.1 is devoted to crystal lattices. With respect to the homonymous Part 9 of ITA5 we notice: (1) a significantly modified table (Table 3.1.2.3) of Delaunay classification, now with the corresponding projections along symmetry directions and Dirichlet (Voronoi) domains in the unit cell; (2) a detailed example of Delaunay reduction; and (3) an extended description of the metric restrictions imposed by symmetry, with emphasis on the limiting cases.

Chapter 3.2 deals with point groups and crystal classes, and is an extensively rewritten text with respect to Part 10 of ITA5. The 47 crystal and point forms are now presented not only in a table but also with the corresponding drawings (the change in the name of point form No. 7, from 'quad' to 'rectangular prism', is appreciated). A major update consists of a new section on molecular symmetry (Section 3.2.4), accompanied by the corresponding Wyckoff positions for point groups; chemists will certainly appreciate this addition.

Chapter 3.3 explains the space-group symbols and their use. It corresponds to Part 12 of ITA5 with a few updates. Chapter 3.4 is devoted to lattice complexes and is a significant update of Part 14 of ITA5. In particular, efforts have been made to clarify the relations and differences between lattice complexes, point configurations and crystallographic orbits. Their kinship often hides the differences and these concepts have often been the cause of some headache for the less-experienced reader. With this revised text, new light should now be shed on this landscape.

Chapter 3.5 inherits the text about normalizers that occupied Part 15 of ITA5, with the addition of a third category of normalizers (besides Euclidean and affine): the chiralitypreserving Euclidean normalizers, i.e. the Euclidean normalizers of Sohncke types of space groups. In the literature we keep on seeing widespread confusion between the chirality of crystal structures (which is possible in any of the 65 Sohncke types of space groups) and that of groups (which concerns the 22 types forming 11 enantiomorphic pairs). A simple way to avoid this confusion is to look at the Euclidean normalizer: the condition for a group to be chiral is that it possesses only a chirality-preserving type of Euclidean normalizer.

The last chapter, 3.6, is brand new and provides an introduction to magnetic subperiodic groups and space groups. The tables of magnetic groups are available in electronic format (Litvin, 2013) and could never be printed as they are too extensive; this chapter gives guidelines on how to read and understand them.

In conclusion, the updates and improvements in this new edition of Volume A are too numerous and important to describe all of them in full detail. Special care has been taken to get a result approachable by the good-willed beginner, while nevertheless keeping the necessary rigour. No doubt every investigator working with condensed matter will greatly benefit by having a copy of this new edition handy on their shelf.

\section{References}

Litvin, D. B. (2013). Magnetic Group Tables. 1-, 2- and 3-Dimensional Magnetic Subperiodic Groups and Space Groups. Chester: International Union of Crystallography. Freely available from http:// www.iucr.org/publ/978-0-9553602-2-0.

Momma, K. \& Izumi, F. (2011). J. Appl. Cryst. 44, 1272-1276.

Nespolo, M. \& Aroyo, M. I. (2016). Acta Cryst. A72, 523-538.

Wondratschek, H. \& Müller, U. (2010). International Tables for Crystallography, Vol. A1, Symmetry Relations Between Space Groups, 2nd ed. Chichester: Wiley.

Wyckoff, R. W. G. (1922). The Analytical Expression of the Results of the Theory of Space-Groups. Washington: Carnegie Institution.

Wyckoff, R. W. G. (1930). The Analytical Expression of the Results of the Theory of Space Groups, 2nd ed. Washington: Carnegie Institution. 